\title{
CFD study of the effect of baffles on the energy consumption and the flow structure in a vessel stirred by a Rushton turbine
}

\author{
Y. Kamla*, M. Bouzit**, A. Hadjeb***, I.M. Arab****, M. Beloudane***** \\ *Université des sciences et de la technologie USTO-MB, 1505 El M'nouar, Oran, Algeria, Laboratoire des sciences et \\ ingénieries maritimes E-mail: youcef.kamla@univ-usto.dz. \\ **Université des sciences et de la technologie USTO-MB, E-mail: bouzit_mohamed@yahoo.fr \\ ***Université des sciences et de la technologie USTO-MB, E-mail:arab_ilies@gmail.com \\ cross $^{\text {ref }}$ http://dx.doi.org/10.5755/j01.mech.22.3.12663
}

\section{Nomenclature}

$a$ - blade width, $\mathrm{m}$; $d s$ - shaft diameter, $\mathrm{m} ; c$ - impeller offbottomed clearance, $\mathrm{m}$; $d$ - impeller diameter, $\mathrm{m} ; b$ - blade height , m; $W$-baffle length, m; $D$-tank diameter, m; $H$ - liquid level, m; $N$-impeller rotational speed, $1 / \mathrm{s}$; $P$-power, $\mathrm{W}, \omega$ - direction of impeller rotation; $\mathrm{Np}$ power number, dimensionless; $R$ - radial coordinate, m; $R^{*}$ - dimensionless radial coordinate, $R^{*}=R / D ; R e$ Reynolds number, dimensionless; $R c^{*}$ - radius of the baffle curvature, dimensionless; $V_{z}$ - axial velocity, $\mathrm{m} / \mathrm{s} ; V_{\theta}$ tangential velocity, $\mathrm{m} / \mathrm{s} ; V_{r}$ - radial velocity, $\mathrm{m} / \mathrm{s} ; Z$ - axial coordinate, $\mathrm{m} ; Z^{*}$-dimensionless axial coordinate, $Z^{*}=2 Z / D ; \rho$ - fluid density, $\mathrm{kg} / \mathrm{m}^{3} ; \mu$ - viscosity, Pa s

\section{Introduction}

When an agitated system is fitted with baffles, a slight increase in the power consumption will be required [1]. On the other hand, the baffles break the tangential flow induced by the rotation of the agitator and they transform it into axial and radial components that obstruct the rotational movement. This resistance results in an increase in the power consumption.

Iranshahi et al. [2] studied experimentally the baffles repercussions on the power consumption and the hydrodynamic characteristics of an agitated tank by a Maxblend impeller. Lu et al. [3] examined by experiments the effect of width and number of baffles (from 2 to 8 baffles) on the mixing time in mechanically agitated vessels with single and triple standard Rushton turbine for a system with and without aeration.

Other authors studied the effects of some geometrical parameters of baffles such us (number, width, length and distance between the lower edge of the baffle and the bottom of the vessel) on the heat transfer coefficient in an agitated tank by a RT (Rushton Turbine), PBT (Pitched Blade Turbine) and a propeller [4].

Vakili and Esfahany [5] studied the effect of baffle width for two-blade impeller. Bittins and Zehner [6], Karcz et al. [7] and Ammar et al. [8] interested to the influence of the baffles length on the power consumption for different kinds of agitators: RT, PBT and a propeller. They noted that the power number strongly depends on the baffles length. Khazam et al. [10] showed the effect of baffles configuration on the drawdown agitator speed and power consumption for the PBT and A340 impellers.

Youcefi et al. [9] studied numerically the effect of three types of a cylindrical tank: with baffles, without baf- fles, and a tank with slots placed at the external perimeter of its vertical wall. They concluded that these slots contribute to the reduction of the power consumption and the vortices size.

An efficient impeller should ensure the fluid circulation in the whole vessel volume. The design of the impeller, its location in the vessel and its rotational speed, the tank design and the fluid properties influence strongly the performance of such mixing systems. Somme authors interested to the effects of the impeller design [11], others to the tank design [13-14], the shaft eccentricity [15-19], the spacing between two impellers [20-24], or the impeller rotational speed [25-27].

In the present paper, we interest to the RT (Rushton Turbine) operating in the turbulent regime with a Newtonian fluid. We focus of the effects of baffles on the flow structure and power consumption. Our search in the literature reveals that no study has been achieved concerning the effect of the baffle curvature. Therefore, we intend to highlight the effect of this parameter on the efficiency of a stirred system.

\section{Presentation of the problem}

The mixing configuration consists of a flat bottomed cylindrical vessel equipped with a Rushton turbine. The vessel has a diameter $D=150 \mathrm{~mm}$ and it is provided with four baffles of width $W=D / 10$ (Fig. 1).

Mixing is achieved by a standard Rushton turbine with six blades of diameter $d=D / 3$ placed at one third of the liquid height $(c=D / 3)$. Values of the impeller blade height and width are $b=D / 5, a=D / 4$ respectively. The liquid (water) height in the tank is equal to the tank diameter $D=H$. In order to investigate the effects of the baffles curvature $\left(R c^{*}\right)$, we've realized five geometrical configurations: $R c^{*}=0,1 / 30,3 / 30,5 / 30$ and $7 / 30$.

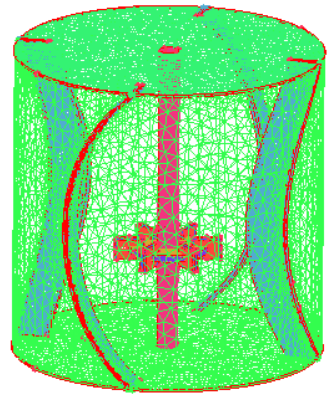

Fig. 1 Mixing configuration

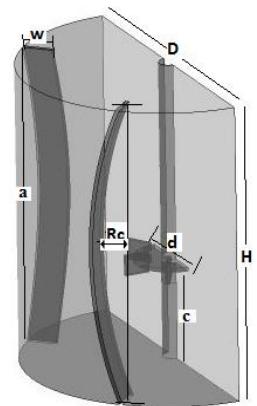

Fig. 2 Stirred system dimension, $R c^{*}=1 / 10$ 
The Reynolds number expressed as follows:

$$
R e=\frac{\rho N d^{2}}{\mu} .
$$
follows:

Results are presented in dimensionless form as

$$
R^{*}=2 R=D ; Z^{*}=\frac{Z}{D},
$$

where $R$ and $Z$ are the radial and axial coordinates, respectively.

The dimensionless velocity and power number are given by the following equations, respectively:

$$
\begin{aligned}
& V^{*}=\frac{V}{\pi N D} ; \\
& N_{p}=\frac{P}{\rho N^{3} d^{5}} .
\end{aligned}
$$

\section{Numerical simulation}

The geometry of the simulated problem is created by the pre-processor INSYS ICEM CFD and then divided into tetrahedral meshes (Fig. 2).

Calculations are achieved by the ANSYS CFX. There are three techniques available to simulate the fluid flows in stirred tanks: the Rotating Reference Frame (approach), the sliding mesh and Multiple Reference Frame (MRF) approach. The last technique is used when the tank is provided with baffles [18, 28, and 29]. In the present paper, the MRF technique is used. In this approach, the area is divided into two zones, a zone around the agitator is simulated in a reference frame which rotates with the stirrer and requires a speed equal to the speed of impeller (rotating frame), the remaining volume of the vessel constitutes the second area wherein the equations are resolved in a fixed reference and given an absolute speed equal to zero (fixed coordinate system). The fluid used is Newtonian (water). The Reynolds number is changed from 40,000 to 60,000 and the standard $(\mathrm{k}-\varepsilon)$ model is used as a turbulence model.

The effect of grid size on the predicted results (power consumption and velocity at the impeller tip) is also studied. A mesh of about 1,232,562 elements has given the best compromise between the accuracy of results and the time required for obtaining convergence. Almost all calculations required about 4,000 to 5,000 iterations and about 4-6 hours of CPU time in a machine Pentium i7 core with $8 \mathrm{Ghz}$ of RAM.

\section{Validation of the predicted results}

The predicted results of the radial velocity were compared with the experimental data done by $\mathrm{Wu}$ and Patterson [30], and the numerical results of Sun et al. [31] and Feng et al. [32] (Fig. 3). Profiles of the axial velocity component were also compared with the numerical results obtained by Youcefi et al. [9], (Fig. 4). The comparison shows a satisfactory accuracy.

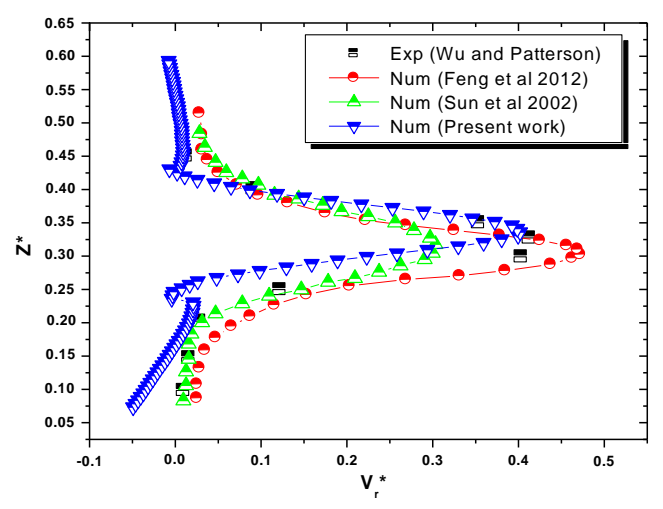

Fig. 3 Radial velocity for $R e=40000, x^{*}=0.22, R c^{*}=0$

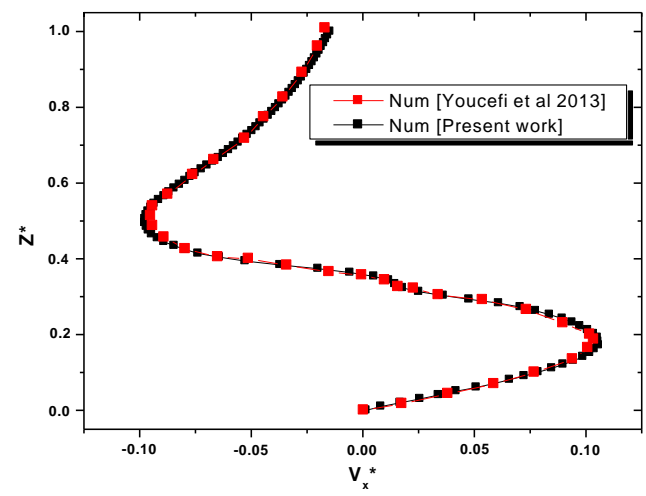

Fig. 4 Axial velocity for $R e=40000, x^{*}=0.266, R c^{*}=0$

\section{Results and discussions}

\subsection{Effect of Reynolds numbers}

Fig. 5 presents the velocity streamlines in a range of Reynolds number varying from 40,000 to 80,000 . The results show the formation of two recirculation loops, one above and other below the impeller. The vortex generated above the impeller increases in size with the increase of Reynolds number.

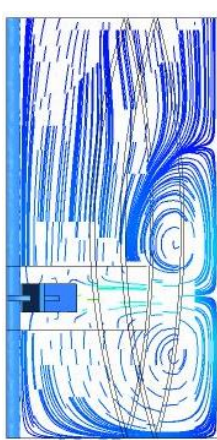

$R e=40000$

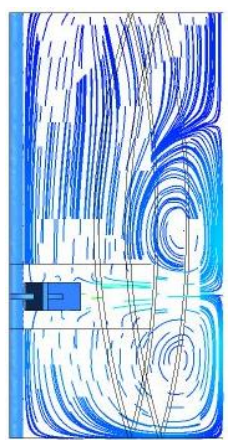

$\operatorname{Re}=60000$

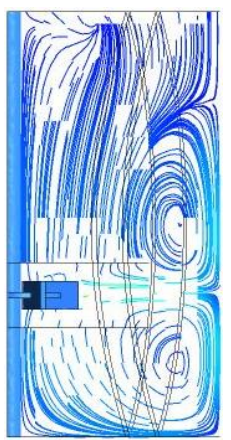

$\operatorname{Re}=80000$
Fig. 5 Velocity stream line for $R_{c} *=1 / 10$, direction of impeller rotation $(-\omega)$

\subsection{Effect of the baffle curvature}

Fig. 6 shows the effects of the baffles curvature on the axial velocity. As remarked, the increase of the baffle curvature in the counter-clockwise direction $(-\omega)$ generates an intense flow near the free surface of fluid and makes it more disturbed. 


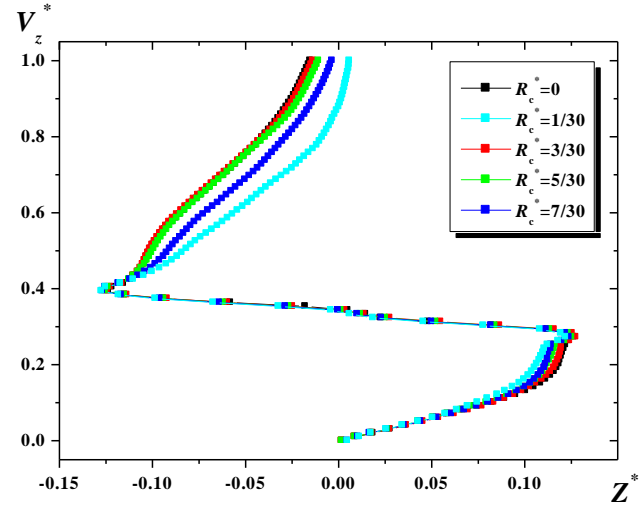

a

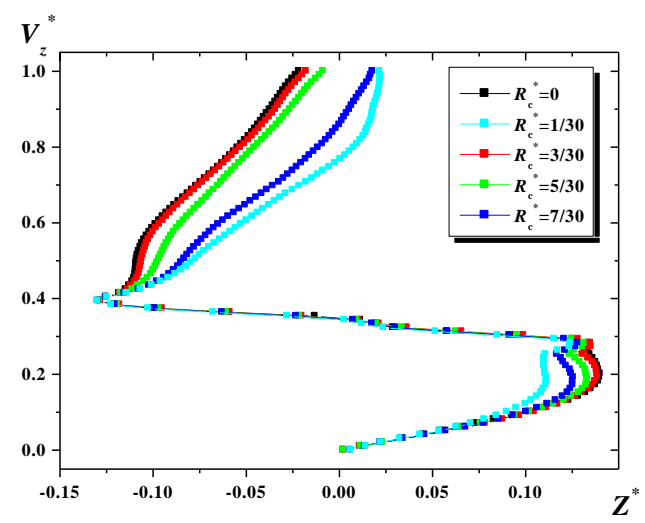

b

Fig. 6 Axial velocity for the impeller rotation $(-\omega), R^{*}=0.2 ; \mathrm{a}-R e=40,000 ; \mathrm{b}-R e=60,000$

5.3. Effect of the baffle curvature and rotational direction

5.3.1. Axial velocity
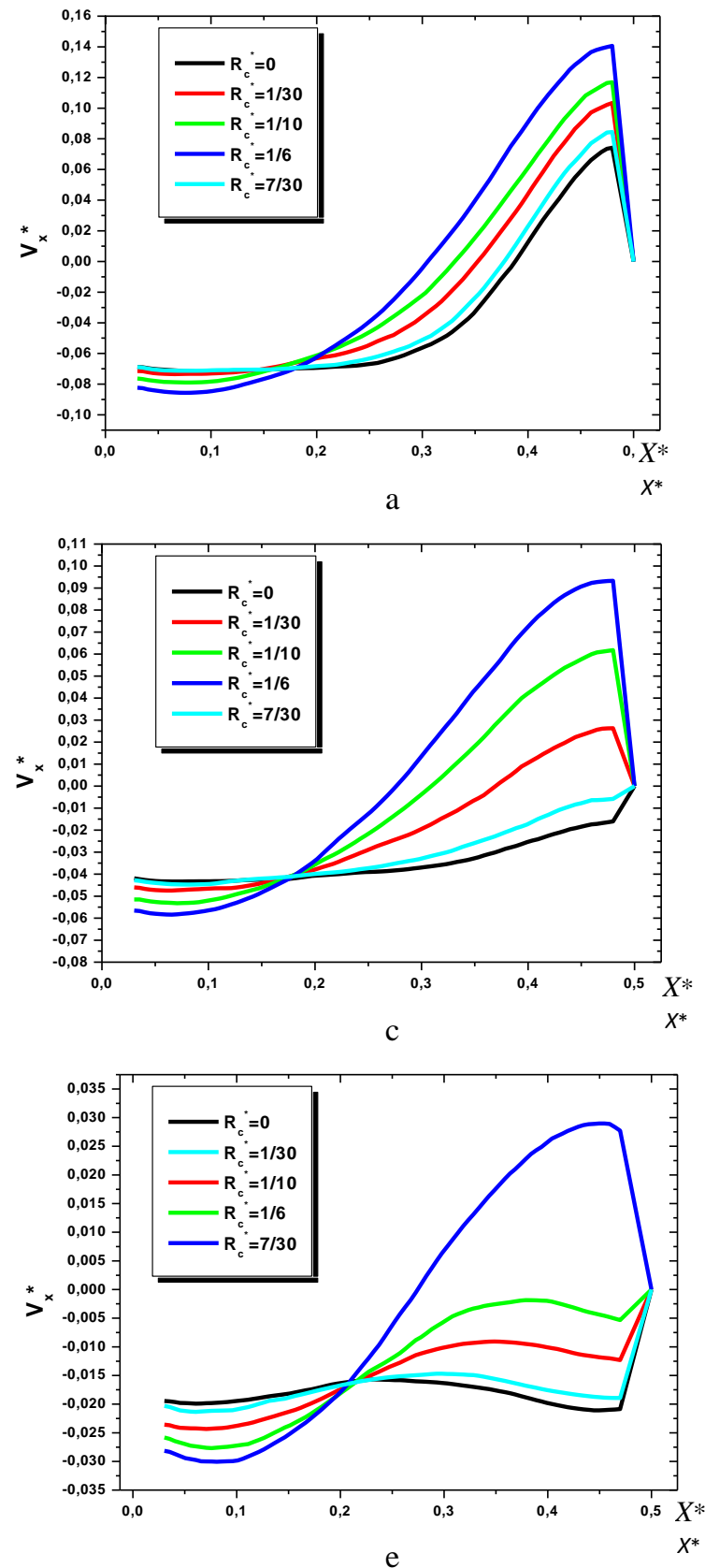
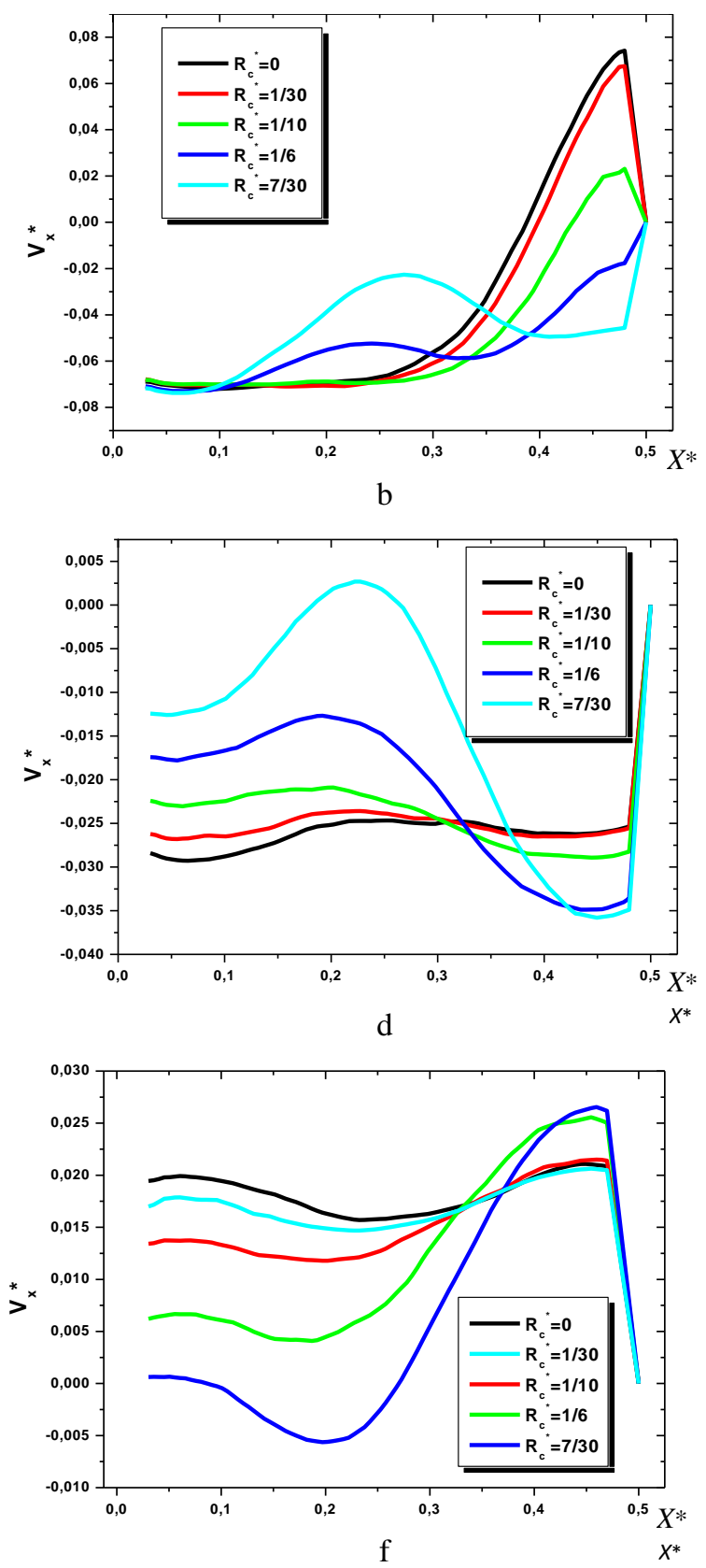

Fig. 7 Axial velocity for $R e=40,000, \theta=0^{\circ}, \mathrm{a}-Z^{*}=0.66,+\omega ; \mathrm{b}-Z^{*}=0.66,-\omega ; \mathrm{c}-Z^{*}=0.9,+\omega ; \mathrm{d}-Z^{*}=0.9,-\omega$; $\mathrm{e}-Z^{*}=0.986,+\omega ; \mathrm{f}-Z^{*}=0.986,-\omega$ 
5.3.2. Flow fields

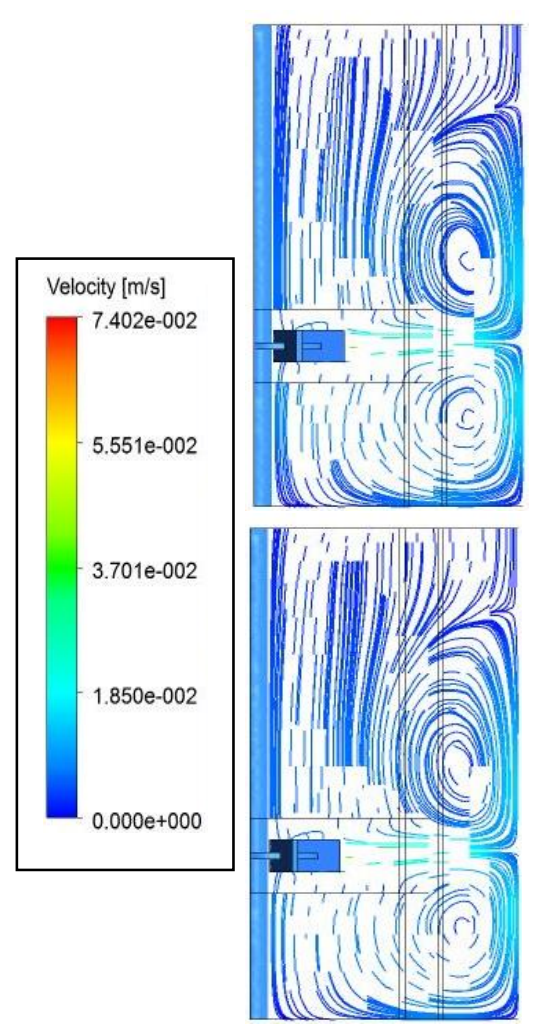

$R_{c}^{*}=0$
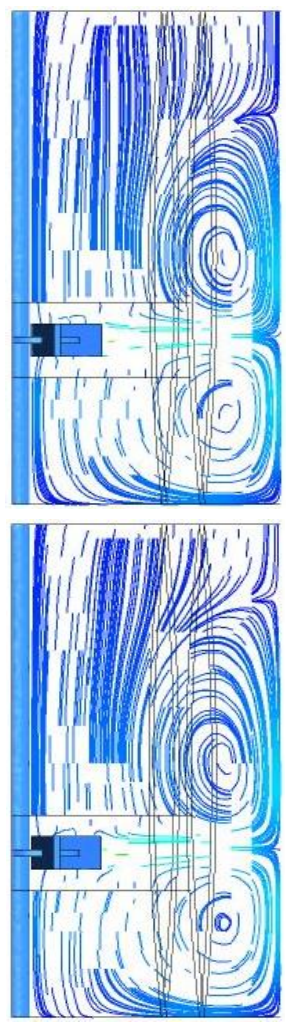

$R_{c}{ }^{*}=1 / 30$
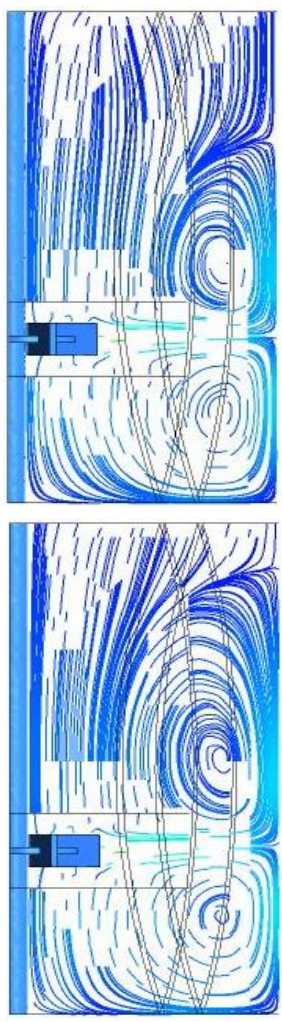

$R_{c}{ }^{*}=3 / 30$

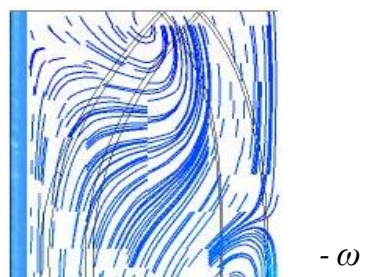

$-\omega$
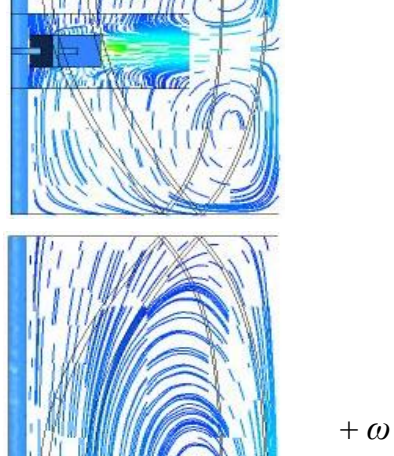

$+\omega$

Fig. 8 Velocity streamlines for $R e=60,000$

Axial velocity profiles at the height $Z^{*}=0.66$ (Figs. 7, a, b) show the vortex formation for all the geometrical arrangements studied and for both rotational directions. The intensity of the axial velocity is more strong for the tank equipped with baffles that have a curvature $R c^{*}=7 / 30$ in the clockwise direction. For the counterclockwise direction $(-\omega)$, the absence of vortices near the free surface for baffled tanks with $R c^{*}=5 / 30$ et $7 / 30$ (Figs. 7, c, d).

For positions near to free liquid surface $Z^{*}=0.986$ (Figs. 7, e, f), the increase of the baffle curvature enhances the axial pumping. The axial velocity is more active for $R c^{*}=R / D=7 / 30$ (i.e. $23 \%$ from the vessel diameter) compared to the other studied cases.
Fig. 8 shows the effects of the geometrical configuration on the flow structure inside the tank for $R e=60,000$. We note that the bending radius of baffles has a great influence on the size of the recirculation loops formed in the upper part of the tank. Indeed, for the counter-clockwise direction $(-\omega)$, the size of the vortices in the upper part is inversely proportional to the baffles curvature radius. However, the relation is proportional for the clockwise direction $(+\omega)$.

An important remark is drawn from Fig. 8 is that the increase of baffle curvature reduces the axial vortices. However and when $R c^{*}=7 / 30$, a secondary vortex is formed for the case of (- $\omega$ ) (as confirmed by Fig. 9).

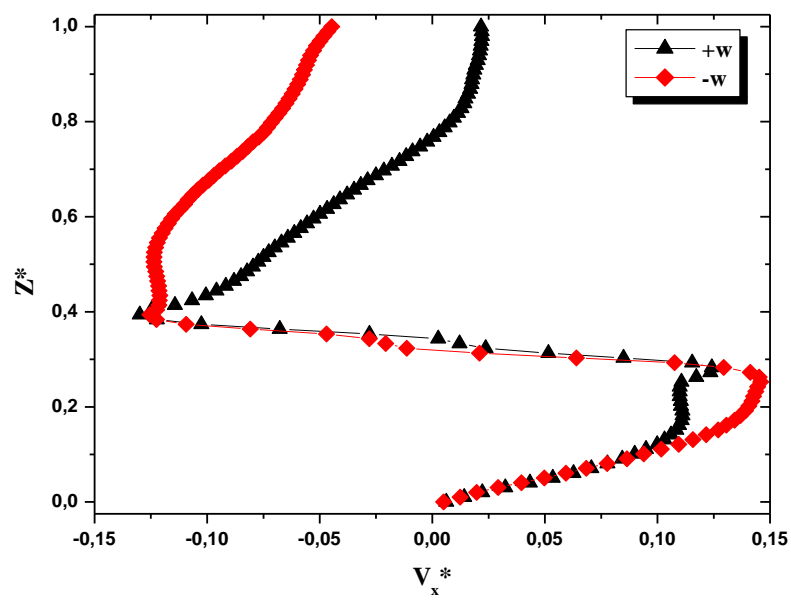

Fig. 9 Axial velocity for $R e=60,000, R^{*}=0.2, R c^{*}=7 / 30$ 

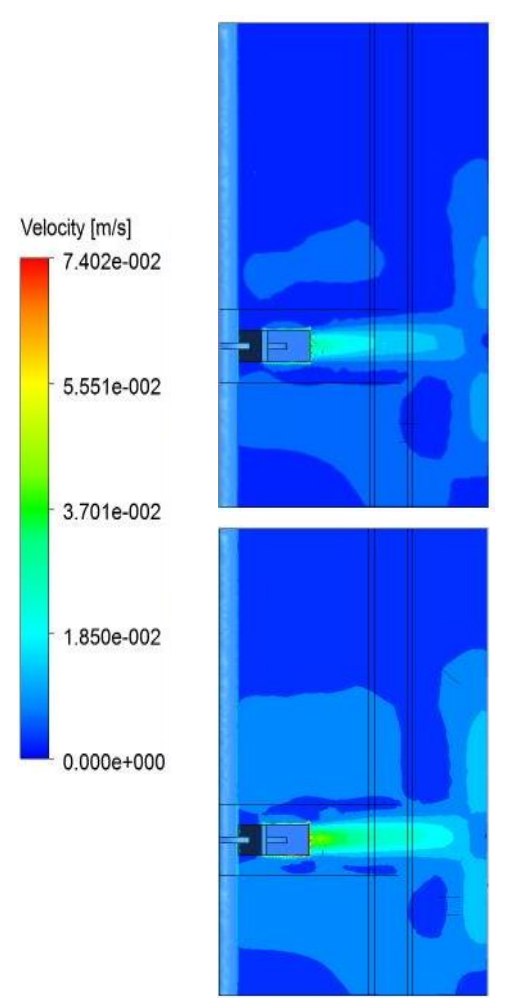

$R_{c}^{*}=0$
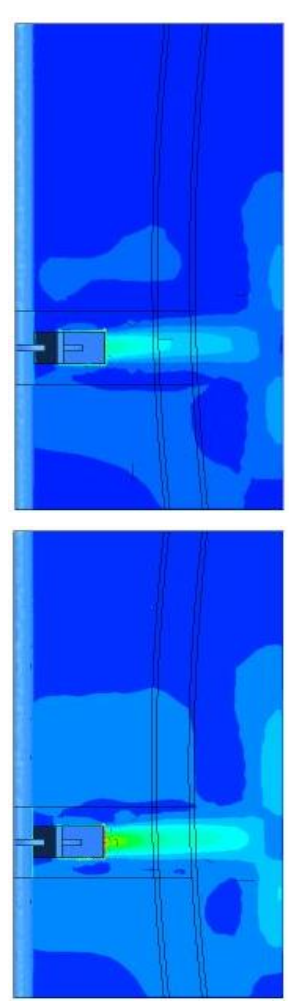

$R_{c}^{*}=1 / 30$
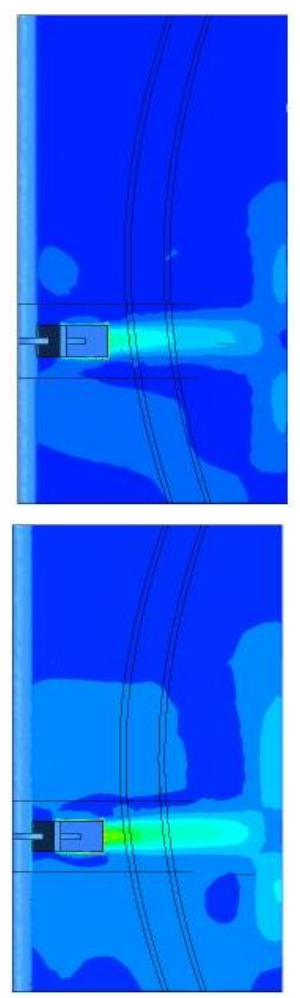

$R_{c}^{*}=3 / 30$

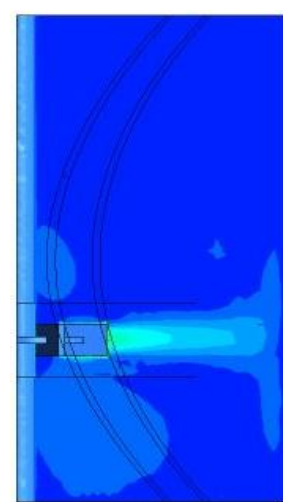

$-\omega$

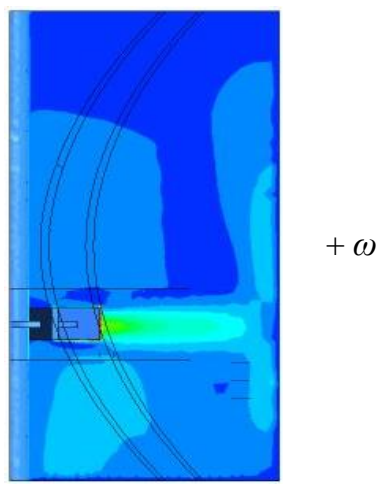

$R_{c}^{*}=7 / 30$

Fig. 10 Velocity contours for $R e=60,000$

Elson et al. [33] demonstrated that the presence of baffles increases considerably the well-stirred region size for both vertical and horizontal directions. Fig. 10 allows us to compare the effects of the impeller rotational direction and the baffles curvature on the well-stirred region size. For the case $(+\omega)$, the results show that the well stirred region size becomes wider while increasing the baffles curvature. We note also that the fluid motion in the lower part of the vessel becomes more intense when the blade is curved by $23 \%$.

The present results prove that the curvature of baffles in the case $(+\omega)$ increases the well-stirred region size even more than straight baffles and therefore increases the mixing efficiency. The case $(+\omega)$ is more efficient than the case $(-\omega)$.

\subsubsection{Vortex size}

The variation of the vortex size (recirculation loop) in the upper part of the vessel with respect to the bending radius of the baffles is presented in Fig. 11.

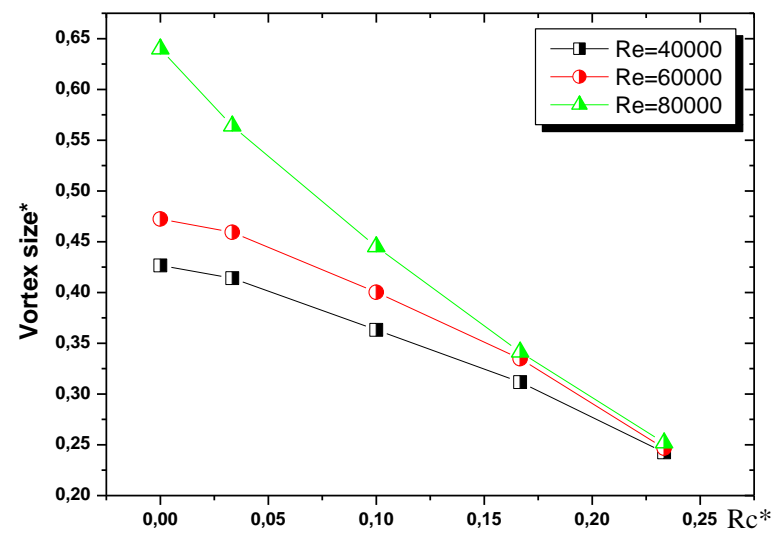

Fig. 11 Vortex size for the case $(-\omega)$

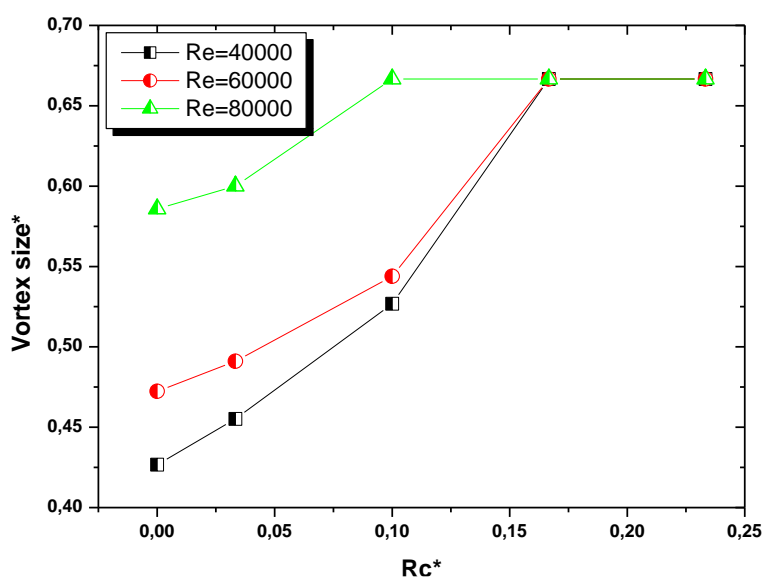

Fig. 12 Vortex size for the case $(+\omega)$

As shown, for the counter-clockwise direction $(-\omega)$, the increase of the baffle curvature the decrease of the vortex size. The opposite fact is noticed for the case $(+\omega)$ where the vortex size is this time proportional to the radius of curvature (Fig. 12). These remarks are valid for the three values of Reynolds numbers $(R e=40,000,60,000$ and 80,000).

\subsubsection{Dead zones behind baffles}

Fig. 13 illustrates the formation of a dead zone behind the straight baffle. When the impeller is rotating in opposite clock-wise direction, the increase of the baffle curvature yields large dead zones. However and in the case of $(+\omega)$, the curvature provides the advantage to eliminate these zones resulting thus in better mixing characteristics. Dead zones are completely disappeared for a bending radius $R c^{*}=3 / 30$ and $7 / 30$ with the rotational direction $(+\omega)$. 


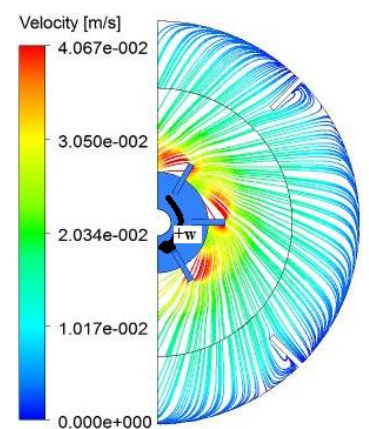

$R_{c} *=0$

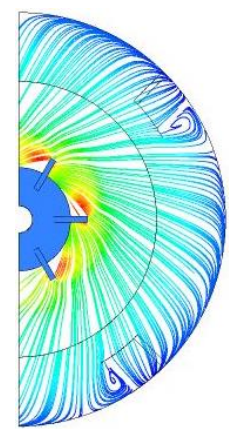

$R_{c} *=1 / 10$

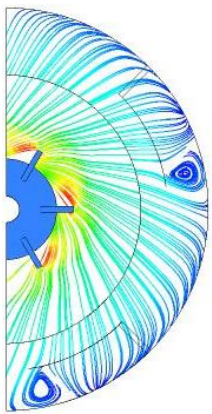

$R_{c} *=7 / 30$

Fig. 13 Formation of dead zones behind the baffles, at $R e=40,000, Z^{*}=0.33,(-\omega)$

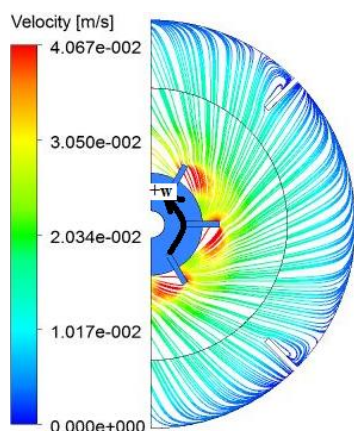

$R_{c}^{*}=0$

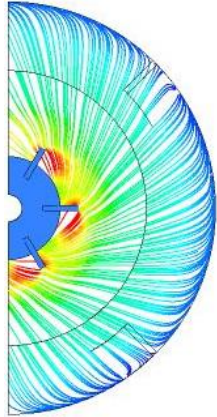

$R_{C} *=1 / 10$

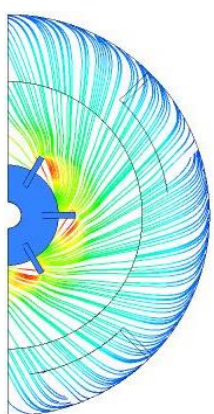

$R_{c}^{*}=7 / 30$

Fig. 13 Formation of dead zones behind the baffles, at $R e=40,000, Z^{*}=0.33,(+\omega)$

\section{Power consumption}

The power consumption in stirred tanks is known as one of the most important parameter which is influenced by several factors such as: impeller and tank design. Given this definition, our attention was focused on the investigation of the baffle curvature effects on the power consumption.

Different comparisons between our predicted results and other experimental and numerical data are provided and summarized in Table. The validation shows a satisfactory agreement.

For the case $(-\omega)$, the power consumption begins to decrease with increasing baffle curvature. However, when the baffle is curved by $10 \%\left(R c^{*}=R c / D=10 \%\right)$, the power consumption increases newly and then it remains almost constant for further increase of $R c^{*}$.

For the case $(+\omega)$, the results presented in Table reveals a continuous reduction in the power consumption with increasing baffle curvature. When $R c^{*}=7 / 30$ (i.e. $23 \%$ from the vessel diameter), the power consumption is reduced by about $47 \%$ compared to the straight baffle.

Table

Power number for $\operatorname{Re}=4 \times 10^{4}$

\begin{tabular}{|c|c|c|c|c|c|c|c|}
\hline & Exp. [34] & Num. [9] & \multicolumn{5}{|c|}{ Num. [Present work] } \\
\hline$R c^{*}$ & 0 & 0 & 0 & $1 / 30$ & $3 / 30$ & $5 / 30$ & $7 / 30$ \\
\hline$+\omega$ & 6.07 & 6,71 & 6.41 & 5.02 & 5.00 & 4.51 & 3.40 \\
\hline$-\omega$ & $/$ & $/$ & 6.41 & 5.48 & 5.77 & 6.67 & 6.70 \\
\hline
\end{tabular}

\section{Conclusions}

For the counter clockwise rotational direction $(-\omega)$, the curved baffle doesn't present changes in the power consumption compared to the standard form. While a third recirculation loop is created in the upper part of the vessel for the curvatures $R c^{*}=5 / 30$ and $R c^{*}=7 / 30$, which can enhance the mixing efficiency but with instabilities in the free surface of liquid.

For the clockwise rotational direction $(+\omega)$ case, the power consumption decreases with respect to the curvature, the vortex and well-stirred region sizes increase slightly which can enhance the mixing efficiency without introducing damaging instabilities in the free surface area.

We finally conclude that the optimal configura- tion in terms of power consumption and flow circulation is the case of $R c^{*}=7 / 30$ and the positive rotational direction $(+\omega)$. A drop by $47 \%$ in the power consumption is obtained by $R c^{*}=7 / 30$ compared to the straight baffle $\left(R c^{*}=0\right)$ with an equivalent mixing result in term of the well-stirred region size.

\section{References}

1. Pharamond, J.C.; Roustan, M.; Roques, H. 1975. Détermination de la puissance consommée dans une cuve aérée et agitée, Chemical Engineering and Science $30: 907-912$. http://dx.doi.org/10.1016/0009-2509(75)80056-X.

2. Hixson, A.W.; Wilkens, G.A. 1933. Performance of Agitators in Liquid-Solid Chemical Systems, Ind.Engng 
Chem. 25(11): 1196-1203.

http://dx.doi.org/10.1021/ie50287a005.

3. Iranshahia, A.; Devalsa, C.; Henichea, M.; Fradettea L. 2007. Hydrodynamics characterization of the Maxblend impeller, Chemical Engineering Science 62: 3641-3653.

http://dx.doi.org/10.1016/j.ces.2007.03.031.

4. Wei-Ming, L.; Hong-Zhang, W.; Ming-Ying, J. 1997. Effects of baffle design on the liquid mixing in an aerated stirred tank with standard Rushton turbine impellers, Chemical Engineering Science 52: 3843-3851. http://dx.doi.org/10.1016/S0009-2509(97)88929-4.

5. Strek, F.; Karcz, J. 1991. Experimental determination of the optimal geometry of baffles for heat transfer in an agitated, Chemical Engineering and Process 29: 165-172. http://dx.doi.org/10.1016/0255-2701(91)85016-H.

6. Klaus, B.; Zehner, P. 1994. Power and discharge numbers of radial-flow impellers. Fluid-dynamic interactions between impeller and baffles, Chemical Engineering and Processing 33: 295-301. http://dx.doi.org/10.1016/0255-2701(94)01011-0.

7. Karcz, J.; Major, M. 1998. An effect of a baffle length on the power consumption in an agitated vessel, Chemical Engineering and Processing 37: 249-256. http://dx.doi.org/10.1016/S0255-2701(98)00033-6.

8. Ammar, M.; Driss, Z.; Chtourou, W.; Abid, M. S. 2011. Effects of baffle length on turbulent flows generated in stirred vessels, Central European Journal of Engineering 1(4): 401-412.

9. Youcefi, S.; Bouzit, M.; Ameur, H.; Kamla, Y.; Youcefi, A. 2013. Effect of some design parameters on the flow fields and power consumption in a vessel stirred by a Rushton Turbine, Chemical and Process Engineering 34 (2): 393-307. http://dx.doi.org/10.2478/cpe-2013-0024.

10. Khazam, O.; Kresta, M.S. 2009. A novel geometry for solids drawdown in stirred tanks, Chemical Engineering Research and Design 87: 280-290. http://dx.doi.org/10.1016/j.cherd.2008.09.013.

11. Ammar, M.; Chtourou, W.; Driss, Z.; Abid, M.S. 2011. Numerical investigation of turbulent flow generated in baffled stirred vessels equipped with three different turbines in one and two-stage system, Energy 36: 50815093. http://dx.doi.org/10.1016/j.energy.2011.06.002.

12. Dan, Taca C.; Paunescu, M. 2001. Power input in closed stirred vessels, Chemical Engineering Science 56: 4445-4450.

http://dx.doi.org/10.1016/S0009-2509(01)00096-3.

13. Ammar, M.; Chtourou, W.; Driss, Z.; Abid, M.S. 2012. Effect of the tank design on the flow pattern generated with a pitched blade turbine, International Journal of Mechanics and Applications 2(1): 12-19. http://dx.doi.org/10.5923/j.mechanics.20120201.03.

14. Montante, G.; Bakker, A.; Paglianti, A.; Magelli, F. 2006. Effect of the shaft eccentricity on the hydrodynamics of unbaffled stirred tanks, Chemical Engineering Science 61: 2807-2814.

http://dx.doi.org/10.1016/j.ces.2005.09.021.

15. Galletti, C.; Brunazzi, E. 2008. On the main flow features and instabilities in an unbaffled vessel agitated with an eccentrically located impeller, Chemical Engineering Science 63: 4494-4505.

http://dx.doi.org/10.1016/j.ces.2008.06.007.
16. Galletti, C.; Pintus, S.; Brunazzi, E. 2009. Effect of shaft eccentricity and impeller blade thickness on the vortices features in an unbaffled vessel, Chemical Engineering Research and Design 87: 391-400.

http://dx.doi.org/10.1016/j.cherd.2008.11.013.

17. Karcz, J.; Cudak, M.; Szoplik, J. 2005. Stirring of a liquid in a stirred tank with an eccentrically located impeller, Chemical Engineering Science 60: 2369-2380. http://dx.doi.org/10.1016/j.ces.2004.11.018.

18. Ameur, H.; Bouzit, M.; Helmaoui, M. 2011. Numerical study of fluid flow and power consumption in a stirred vessel with a SCABA 6SRGT impeller, Chemical and Process Engineering 32(4): 351-366. http://dx.doi.org/10.2478/v10176-011-0028-0.

19. Rutherford, K.; Mahmoudi, S.M.; Lee, K.S.; Yianneskis, M. 1996. The Influence of Rushton impeller blade and disc thickness on the mixing characteristics of stirred vessels, Transactions of IChemE 74: 369-378.

20. Khopkar, A.R.; Tanguy, P.A. 2008. CFD simulation of gas--liquid flows in stirred vessel equipped with dual Rushton turbines: influence of parallel, merging and diverging flow configurations, Chemical Engineering Science 63: 3810-3820.

http://dx.doi.org/10.1016/j.ces.2008.04.039.

21. Hiraoka, S.; Kato, Y.; Tada, Y.; Ozaki, N. 2001. Murakami Y, Lee Y. S, Power consumption and mixing time in an agitated vessel with double impeller, Trans IChemE 79(A): 805-810.

22. Vasconcelos, J.M.T.; Barata, J.M.; Alves, S.S. 1996. Transitional mixing in multiple-turbine agitated tanks, The Chemical Engineering Journal 63: 53-58. http://dx.doi.org/10.1016/0923-0467(95)03072-7.

23. Markopoulos, J.; Kontogeorgaki, E. 1995. Vortex depth in unbaffled single and multiple impeller agitated vessels, Chemical Engineering Technology 18: 68-74. http://dx.doi.org/10.1002/ceat.270180113.

24. Bhattacharya, S.; Hebert, D.; Kresta, S.M. 2007. Air entrainment in baffled stirred tanks, Chemical Engineering Research and Design, 85(5A): 654-664. http://dx.doi.org/10.1205/cherd06184.

25. Torré, J.P.; Fletcher, D.F.; Thierry, L.; Xuereb, C. 2007. Single and multiphase CFD approaches for modelling partially baffled stirred vessels: Comparison of experimental data with numerical predictions, Chemical Engineering Science 62: 6246-6262. http://dx.doi.org/10.1016/j.ces.2007.06.044

26. Assirelli, M.; Bujalski, W.; Eagleshamb, A.; Nienow, A.W. 2008. Macro- and micro mixing studies in an unbaffled vessel agitated by a Rushton turbine, Chemical Engineering Science 63: 5-46. http://dx.doi.org/10.1016/j.ces.2007.07.074.

27. Wu, H.; Patterson, G.K. 1989. Laser-Doppler measurements of turbulent flow parameters in a stirred mixer, Chemical Engineering Science 44: 2207-2221. http://dx.doi.org/10.1016/0009-2509(89)85155-3.

28. Sun, H.Y.; Wang, W.J.; Mao, Z.S. 2002. Numerical simulation of the whole three-dimensional flow in a stirred tank with anisotropic algebraic stress model, Chin. J. Chem. Eng. 10: 15-24.

29. Feng, X.; Cheng, J.; Li X.; Yang, C.; Mao, Z.S. 2012. Numerical simulation of turbulent flow in a baffled stirred tank with an explicit algebraic stress model, Chemical Engineering Science 69: 30-44. http://dx.doi.org/10.1016/j.ces.2011.09.055. 
30. Elson, T.P.; Cheesman, D.J.; Nienow, A.W. 1986. Xray studies of cavern sizes and mixing performance with fluids possessing a yield stress, Chem. Eng. Sci.41: 25552562. http://dx.doi.org/10.1016/0009-2509(86)80041-0.

31. Rushton, J.H.; Costich, E.W.; Everett, H.J. 1950. Power characteristics of mixing impellers-Part II, Chemical Engineering Progress 46: 467-476.
Y. Kamla, M. Bouzit, A. Hadjeb, I.M. Arab, M. Beloudane

CFD STUDY OF THE EFFECT OF BAFFLES ON THE

ENERGY CONSUMPTION AND THE FLOW

STRUCTURE IN A VESSEL STIRRED BY A RUSHTON TURBINE

S u m m a r y

Baffles in stirred tanks provide the considerable advantage of the free surface stability that enhances the turbine's shaft longevity, but they increase the energy consumption due to the flow obstruction that they introduce. So the main issue of the present study is to find a balance between both advantages and disadvantages of baffles. To do so, we investigate numerically the influence of two parameters on the power consumption: the radius of the baffle curvature $\left(R c^{*}\right)$ and the impeller rotational direction. $R c^{*}$ is varying from 0 to $7 / 30$. Results are obtained via the ANSYS CFX code; the Navier-Stokes equations are coupled to the standard $\mathrm{k}-\varepsilon$ turbulence model and solved with the Multiple Reference Frames (MRF) method.

Keywords: Stirred tanks, Power consumption, Baffles, Rushton turbine, Vortex. 